\title{
INOVAÇÃO E IMITAÇÃO NA INDÚSTRIA FARMACÊUTICA: estratégias empresariais a partir da regulamentação dos medicamentos genéricos
}

\author{
Innovation and imitation in the pharmaceutical industry: business \\ strategies facing the generic drugs market regulation
}

\author{
Marco Aurélio Lima de Queiroz ${ }^{a}$, Flávio Carvalho de Vasconcelos ${ }^{\mathrm{b}}$ \\ a Professor FGV/EAESP.São Paulo, SP - Brasil, e-mail: marcoqueiroz@gvmail.br \\ b Professor FGV/EAESP.São Paulo, SP - Brasil, e-mail: flavio.vasconcelos@fgv.br
}

\begin{abstract}
Resumo
$\mathrm{Na}$ indústria farmacêutica, os medicamentos genéricos surgiram como instrumento para propiciar maior competição, reduzindo as imperfeições de mercado inerentes às características e dinâmica daquela indústria. Desenhados para competir fundamentalmente por preço, através da garantia de intercambialidade destes produtos com os medicamentos de referência nos quais se baseiam, constituem um incentivo a um modelo de negócios baseado em eficiência operacional, em imitação e não em inovação. Paradoxalmente, à primeira vista, empresas que se desenvolveram como fabricantes de genéricos começaram a investir em pesquisa e desenvolvimento, e empresas baseadas em inovação estabeleceram operações para fabricação e/ou comercialização de genéricos. Neste trabalho pretende-se contextualizar este cenário, compreender a dinâmica de competição envolvida, e tecer breves considerações sobre as estratégias empresariais adotadas à luz de modelos teóricos sobre estratégia empresarial e sobre inovação.
\end{abstract}

Palavras-chave: Estratégia empresarial; Inovação; Indústria farmacêutica; Medicamentos genéricos.

\begin{abstract}
The market regulation for generic drugs is intended to increase competition, reducing market imperfections typically found in the pharmaceutical industry. Generic drugs are supposed to compete exclusively in price, by the condition of interchangeability of these products with their referred original product. Thus, there is an incentive for firms to adopt a business model based on operational efficiency, not in innovation. It may look like a contradiction or a non-sense business strategy that large generic manufactures started to invest on research and development,
\end{abstract}


and that firms well-known as innovation labs have set up large operations to produce generic drugs. In this work, we intend to set the context of this competition and provide a possible explanation for the competition strategies and dynamics observed, taking into account various theoretical frameworks of business strategy and innovation.

Keywords: Business strategy; Innovation; Pharmaceutical industry; Generic drugs.

\section{INTRODUÇÃO}

Os medicamentos genéricos foram introduzidos em diversos países desenvolvidos após a segunda guerra mundial, sob variadas legislações e sistemas de saúde públicos e privados (COSTA; SILVA; RIBEIRO, 2001). No Brasil, pode-se considerar que o processo de regulamentação dos genéricos, notadamente no período entre 1999 e 2002, envolveu expressiva disputa de interesses políticos e econômicos (DIAS, 2003; FRANÇA, 2005), alcançando grande repercussão na mídia e, provocando juntamente com outros acontecimentos, a instalação de uma Comissão Parlamentar de Inquérito que investigou a conduta dos laboratórios farmacêuticos durante o referido processo (CPI, 2000).

Embora diversos estudos acadêmicos tenham se debruçado sobre o impacto dos medicamentos genéricos em termos de preços (NISHIJIMA, 2003; VIEIRA; ZUCHI, 2006), pouco se investigou sobre o impacto que provocaram na conduta estratégica das empresas farmacêuticas que aqui operavam, salvo exceções (DI BAT'TISTI, 2005). Neste trabalho, empregando-se como estratégia de pesquisa a análise documental. Em perspectiva longitudinal, procurou-se caracterizar, em linhas gerais, a dinâmica de inovação e imitação na indústria farmacêutica, a partir do advento dos genéricos e das respostas estratégicas adotadas pelas empresas farmacêuticas (tanto em âmbito mundial como nacional).

O trabalho desenvolve, ainda que de forma ampla e genérica, um esforço de caracterização de condutas estratégicas das empresas a partir de conhecidas vertentes de estratégia empresarial (Organização Industrial-OI, Visão da Firma Baseada em Recursos-RBV e Capacidades Dinâmicas) e de modelos teóricos de inovação (DOZI, 1988; PAVITT, 1984; TEECE, 1986; AFUAH, 1998); o caso empírico escolhido é percebido como revelando uma dinâmica em que as estratégias de inovação e de imitação são adotadas tanto por laboratórios inovadores como por laboratórios inicialmente dedicados à fabricação de cópias de medicamentos (medicamentos genéricos). $\mathrm{O}$ que pode parecer paradoxal, à primeira vista, revela-se como resposta estratégica (intencional ou emergente) face aos diferentes dilemas que os laboratórios enfrentam.

\section{Medicamentos genéricos para promover competição}

A indústria farmacêutica, em termos globais, vem crescendo de forma sustentada; dobrou de tamanho nos últimos sete anos, alcançando US $\$ 602$ bilhões de vendas anuais de medicamentos em 2005 (IMS HEALTH, 2006), e o Brasil ocupa a posição de 10 ,maior mercado, com vendas de medicamentos de aproximadamente US\$ 9 bilhões (REVISTA GRUPEMEF, 2006).

Este setor apresenta características estruturais de concorrência monopolística de produtos e de oligopólio de empresas (ROMANO; BERNARDO, 2001; SILVA, 2004), o que explica seu invejável retorno econômico $(25 \%$ de margem operacional nas grandes farmacêuticas, contra 15\% em geral na indústria de bens de consumo, segundo BASTOS, 2005). Diversos mecanismos de regulação de preços foram desenvolvidos na grande maioria dos países, dadas as externalidades sociais que a atuação de organizações privadas, na busca de maximização de lucro, inevitavelmente causa (BASTOS, 2005; MARQUES, 2002; ROMANO, BERNARDO, 2001; MELLO, 2001).

O acesso a medicamentos é considerado como um direito assegurado do cidadão (ORGANIZAÇÃO MUNDIAL DE SAÚDE, 2004; BRASIL, 1988), ainda que sua descoberta ou desenvolvimento, produção e comercialização sejam 
atividades em sua maior parte conduzidas por organizações privadas com fins lucrativos. Cabe, portanto, ao Poder Público articular os meios para assegurar a assistência farmacêutica. Neste sentido, justifica-se uma vez mais a preocupação com a regulação deste setor, especialmente perante um quadro de crescentes dispêndios com saúde pública, tanto no Brasil como no resto do mundo (COSTA; SILVA; RIBEIRO, 2001).

O estudo sobre inovação torna-se particularmente interessante nesta indústria; ela é considerada extremamente dinâmica e com grande facilidade para articular e/ou difundir globalmente suas operações industriais e comerciais (FRENKEL, 2002), e historicamente apresenta níveis bastante elevados de investimento de empresas em pesquisa e desenvolvimento, em torno de $14 \%$ das vendas líquidas anuais das grandes farmacêuticas, segundo a IFPMA, 2004.

No final da década de 1990 dois importantes acontecimentos afetaram expressivamente os rumos desta indústria no Brasil, no que diz respeito ao tema objeto deste trabalho: - a regulamentação das patentes (Lei 9.279 de 14.05.96 - BRASIL, 1996) e a regulamentação dos medicamentos genéricos (Lei 9.787 de 10.02.99 - BRASIL, 1999). ${ }^{1}$ Se, por um lado, o reconhecimento das patentes representa um importante incentivo à pesquisa por parte de agentes privados (INTERFARMA, 2006), por outro, a regulamentação dos genéricos estimula um modelo de negócios baseado na exploração de nichos de mercado já maduros, cujos medicamentos de referência estejam com patentes vencidas ou próximas de expirar.

É importante considerar que no Brasil, até então, a ausência de patentes para produtos farmacêuticos estimulara uma indústria nacional fundamentalmente baseada em cópias de produtos bem sucedidos, produzindo os chamados "similares". (FRENKEL, 2002). Competindo com os produtos de referência, no entanto, os similares não haviam alcançado muita confiança da classe médica ou dos consumidores, salvo em alguns casos em que desenvolveram efetivamente uma marca própria. A dúvida quanto à qualidade destes medicamentos era sustentada pela existência de centenas de laboratórios "de fundo de quintal" que importavam matérias-primas baratas, de origem e qualidade duvidosa, somada à falta de aparelhamento da vigilância sanitária para a fiscalização destes agentes econômicos e da cadeia de distribuição na qual se encontravam inseridos. ${ }^{2}$

Ao introduzir os medicamentos genéricos, com o conceito de intercambiabilidade, isto é, a obrigação de que os medicamentos comercializados como genéricos comprovassem o mesmo efeito terapêutico que os medicamentos de referência, podendo substituí-los com a mesma eficácia, criou-se um grande estímulo para que laboratórios privados desenvolvessem um modelo de negócios baseado em imitação, com eficiência e competitividade de custos.

Pode-se afirmar, com base no comportamento da maioria das grandes empresas farmacêuticas (big pharma), que o mercado de medicamentos genéricos foi interpretado predominantemente como uma ameaça e não como uma oportunidade; as margens de lucro elevadas com medicamentos consagrados, já fora do período de proteção patentária, constituíam importante fonte de geração de caixa que, segundo as empresas, era vital para sustentar os elevados patamares de investimento de longo prazo em pesquisa e desenvolvimento (IFPMA, 2004; INTERFARMA, 2006). São evidências para esta assertiva as investigações e/ou condenações por prática anticoncorrencial relacionada com os medicamentos genéricos, envolvendo grande número de laboratórios, tanto no Brasil como nos EUA. ${ }^{3}$ Estudo longitudinal de Trullen e Stevenson (2006) sobre o

\footnotetext{
${ }^{1}$ Considera-se o Decreto 793 , publicado em 06/04/93 como a primeira tentativa de regulamentação dos medicamentos genéricos que foi rechaçada por inúmeras ações na justiça, impetradas pela indústria farmacêutica; dentre outros aspectos, o Decreto propunha o destaque obrigatório da denominação genérica em todos os medicamentos e a prescrição pela denominação genérica. Não abordava, contudo, a questão da intercambialidade, elemento central à caracterização posteriormente dada a medicamentos genéricos.

2 O reconhecimento dos "laboratórios de fundo de quintal” é feito expressamente nas declarações de José Eduardo Bandeira de Mello, presidente da Associação Brasileira da Indústria Farmacêutica - ABIFARMA na revista RADIS Súmula, abril, 1995 e de Sara Kanter, diretora da Associação dos Laboratórios Farmacêuticos Nacionais - ALANAC na revista RADIS Tema, maio de 2000. Collusion to stall Generics is subject of FTC probe, Chemical Market Reporter, 23.10.00 nos EUA; Condenação de vinte laboratórios pelo CADE - Ministério da Justiça, em 2005 (processo administrativo n.08012.009088/1999-48).
} 
comportamento de sete grandes laboratórios face às ameaças de quebra de patentes e licenciamento compulsório para fabricação de drogas contra a AIDS no atendimento da população africana, descreve algumas das estratégias empregadas.

Embora o mercado de medicamentos genéricos tenha assumido uma grande proporção em termos mundiais, como será visto logo adiante, o volume de investimentos em pesquisa e desenvolvimento na indústria farmacêutica seguiu crescendo de forma expressiva US $\$ 39.4$ bilhões somente por companhias americanas em 2005, US $\$ 32$ bilhões em 2002, contra cerca de US\$200 bilhões na década anterior (PHRMA, 2003). Diversas empresas focadas em inovação decidiram competir também com genéricos. Novas empresas dedicadas a genéricos cresceram extraordinariamente e, curiosamente, começam a se voltar também para inovação. É sobre esta dinâmica que se pretende aqui discorrer, observando particularidades e conseqüências no caso brasileiro, e interpretando as estratégias empresariais adotadas à luz de modelos teóricos de estratégia e de inovação.

\section{A janela de oportunidade para novos entrantes}

A regulamentação dos medicamentos genéricos, no Brasil e em muitos outros países, afetou significativamente o cenário competitivo e o desempenho econômico das empresas farmacêuticas, nacionais e internacionais.

No mercado internacional, os genéricos já alcançaram 53\% das prescrições nos EUA em 2005 (IMS HEALTH, 2006), representando vendas anuais de mais de US\$19 bilhões. A região da Ásia-Pacífico responde por US\$ 14 bilhões em vendas anualizadas e o mercado europeu de Genéricos está estimado em US\$ 11 bilhões, com destaque para Alemanha e Inglaterra (DATAMONITOR, 2005b). O impacto sobre o desempenho econômico esperado das big pharma foi significativo. Nos últimos cinco anos, o valor de mercado das principais indústrias farmacêuticas listadas nas bolsas de valores de Nova York e Frankfurt apresentou redução média de 21\%. A concorrência com os genéricos é apontada como uma das principais causas desta deterioração nas expectativas de lucro no longo prazo. (TENDENCIAS CONSULTORIA INTEGRADA; 2006a, 2006b). As perspectivas seguem sendo de crescimento "meteórico" para o mercado de medicamentos genéricos (DATAMONITOR, 2005a). Patentes a expirar devem abrir mercados de mais de US\$ 20 bilhões em 2006; 80\% dos medicamentos sob prescrição "campeões de vendas" em 2003 irão expirar até 2010, representando, somente eles, um mercado potencial de US\$ 110 bilhões, segundo estimativas (CHEMICAL MARKET REPORTER, 2006).

Apostando na capacidade de manter pipelines de novas drogas promissoras, as grandes farmacêuticas, quase sem exceção, haviam reafirmado seu foco no mercado de produtos éticos e sob proteção de patentes (venda sob prescrição com indicação da marca), mesmo sob a pressão dos genéricos. Em 2000, a Chemical Market Reporter já apontava a necessidade destas firmas desenvolverem estratégias de defesa robustas. Veremos que diversas delas, não sem alguma hesitação, que lhes custou perda de market share, decidiram investir também fortemente no negócio dos genéricos.

Fundamental para a dinâmica de inovação que se pretende analisar neste trabalho, foi o surgimento de novos laboratórios, percebendo a janela de oportunidade aberta com a crescente regulamentação e incentivo, em âmbito mundial, ao consumo dos medicamentos genéricos (MARQUES, 2002). Estas novas empresas alcançaram posições de destaque no mercado nacional ou global, sem terem desenvolvido qualquer medicamento inovador, dedicando-se à fabricação e comercialização de medicamentos genéricos, concorrendo em preços com os medicamentos de referência (marcas conhecidas com patentes não reconhecidas ou vencidas). Competidores internacionais relevantes surgiram em países antes periféricos na indústria farmacêutica, como o Canadá (Apotex), a Índia (Ranbaxy, Dr.Reddy's), Israel (Teva) e mesmo na China (Wuhan), notadamente por conta da legislação naqueles países, que não reconhecia até então o direito de patentes ou permitia o licenciamento compulsório.

No mercado nacional, os genéricos já respondem hoje por $13,87 \%$ de participação de mercado em unidades (ASSOCIAÇÃO PRÓ- 
GENÉRICOS, 2006), 18,47\% do receituário nas classes terapêuticas onde competem, segundo estimativa (REVISTA GRUPEMEF, 2006). A promoção dos genéricos constituiu uma estratégia central do governo no período de 1999 a 2003 na área da saúde, desde a promulgação da referida lei, envolvendo um conjunto de regulamentações subseqüentes, notadamente até 2003, visando remover obstáculos e inibir condutas de agentes econômicos que pudessem comprometer seu crescimento (ANVISA, 2006; DIAS, 2003).

EMS-Sigma, Medley, Eurofarma são destaques de laboratórios nacionais que, percebendo este mercado como uma grande oportunidade, adotaram uma postura agressiva e alcançaram uma expressiva participação de mercado. Somadas, as três empresas detinham, em 2004, cerca de $60 \%$ do mercado de genéricos, segundo dados do IMS HEALTH, publicados em 29 de junho de 2005 na revista exame (REVISTA EXAME, 2006). A investida dos laboratórios nacionais no mercado de genéricos pode ser percebida pela disparidade ao comparar o número de registros de medicamentos genéricos solicitados pelos diversos laboratórios: EMS-Sigma detinha em agosto de 2006 um total de 408 registros, Medley 143 e Eurofarma, 138. A título de comparação, Ranbaxy contava com 89, Apotex com 48, Novartis (Sandoz) com 53 e Merck 44, segundo informações da ANVISA (2006). Em função do crescimento em genéricos, as empresas nacionais estão aumentando paulatinamente sua participação no mercado farmacêutico brasileiro, historicamente situada abaixo de 30\% (FRENKEL, 2002; QUEIROZ; GONZALES, 2001).

\section{Escolhas estratégicas face às diferenças entre os modelos de inovação e imitação}

Deve-se atentar para as diferenças importantes entre dois modelos de negócios que, para fins analíticos deste trabalho, denominou-se inovação e imitação. Enquanto as big pharma caracterizavam-se como empresas orientadas à inovação, com um modelo de negócios fundamentado na pesquisa e desenvolvimento, com marketing farmacêutico dirigido primariamente ao prescritor, investindo 35 a $50 \%$ de suas vendas líquidas nestas atividades (BASTOS, 2005;
FRENKEL, 2002; QUEIROZ; GONZALES, 2001; ROMANO; BERNARDO, 2001), os fabricantes de genéricos dedicam seus esforços a uma arquitetura eficiente de sua cadeia de produção e na conquista dos pontos de venda e distribuição. Uma evidência interessante, neste sentido, no Brasil, é a concentração de plantas industriais modernas para a fabricação de genéricos em Anápolis (GO), considerando vantagens de custo de mão-de-obra, custos logísticos e benefícios fiscais, aspectos secundários no modelo de inovação, uma vez que os custos de produção neste modelo não respondem por mais de $20 \%$ do preço do medicamento.

É importante salientar que o processo de fabricação de medicamentos não é complexo; máquinas e equipamentos utilizados podem ser facilmente adquiridos, e não há economias de escala expressivas envolvidas. Quanto às matérias-primas (fármacos) necessárias ao atendimento da demanda atual por medicamentos no Brasil, grande parte delas não está mais sob a proteção de patentes, podendo ser encontrada no mercado internacional. Eventualmente, os próprios fármacos poderiam ser produzidos localmente, como vinha acontecendo no Brasil no início da década de 90, antes da "desindustrialização" desse segmento produtivo, pela combinação de um processo de abertura comercial, falta de incentivos à produção nacional e estratégias de globalização empregadas pelas big farma (FRENKEL, 2002; MAGALHÃES et al., 2003; MARQUES, 2002; NEGRI; GIOVANNI, 2001).

Assim sendo, se o principal atributo de um genérico, em tese, é justamente o seu caráter genérico, de intercambiabilidade de produtos, a competição por custos será implacável. Considerase que a rentabilidade esperada (margem de lucro bruta) com a descoberta de novas entidades químicas está entre $60 \%$ a $100 \%$, enquanto que para produtos genéricos situa-se entre 12 a $20 \%$ (BARTLETT, 2000). O mercado de genéricos cresce de forma impressionante mas os competidores também. Em termos teóricos, no limite de uma competição perfeita, esta atividade econômica deixará de ser atrativa (BESANKO; DRANOVE; SHANLEY et al., 2000).

À luz dos referenciais teóricos predominantes em estratégia, a competição dos medicamentos genéricos colocou as grandes farmacêuticas em face de um dilema: como prosseguir no core business e, ao mesmo tempo, 
sustentar ou ampliar sua participação de mercado, competindo com genéricos? Os fabricantes de genéricos, por sua vez, têm que resolver a questão: como sustentar o desempenho econômico no longo prazo com um modelo de negócios baseado na imitação? Considerando as vertentes teóricas predominantes no campo da Estratégia Empresarial (RAMOS-RODRIGUEZ; RUÍZ-NAVARRO, 2004), desenvolvem-se a seguir, sem pretensão de um esforço exaustivo, possíveis respostas das empresas na busca de um desempenho econômico superior sustentado, ou vantagem competitiva, nos termos que a definem Vasconcelos e Cyrino (2000) ou Besanko et al. (2000).

Tomando-se como ponto de partida a perspectiva da Organização Industrial - OI (CAVES, 1980; CAVES; PORTER, 1977; PORTER, 1980, 1985), observa-se que os medicamentos genéricos afetam severamente o potencial de obtenção de "rendas de monopólio", fonte da vantagem competitiva das empresas: as barreiras à entrada de concorrentes e de produtos substitutos foram substancialmente removidas para produtos com patentes vencidas; a alternativa mais plausível, possivelmente, seria a atuação em nichos que valorizam ainda a marca; a estratégia principal dos laboratórios inovadores, de diferenciação, não deveria ser alterada. Tentar diferenciar-se e ao mesmo tempo competir em custos não é possível; eventualmente dois negócios separados poderiam buscar fazê-lo. No negócio de genéricos, seria preciso alcançar a posição de liderança em custos.

Ao considerar como referência a Visão da Firma Baseada em Recursos - RBV (BARNEY, 1991, 1986; PETERAF, 1993), é interessante notar que os fabricantes de genéricos não parecem possuir, a priori, recursos que possam ser considerados raros, valiosos, difíceis de imitar ou substituir. Assim, a obtenção de um desempenho econômico superior sustentado precisaria ser buscada no modelo de inovação, onde estruturas de pesquisa e desenvolvimento, patentes, relacionamento com a classe médica, dentre outros, constituem possíveis fontes de rendas econômicas ("rendas ricardianas"). A marca e/ ou a reputação certamente podem vir a constituir fontes de vantagem competitiva, mas parece arriscado investir nela para competir em um mercado que se propõe "genérico". Uma possível alternativa para fabricantes de genéricos seria a obtenção de patentes, mediante acordos de licenciamento, com empresas voltadas para pesquisa. Mas certamente as margens seriam reduzidas, pois a capacidade de manufatura e distribuição não constitui, salvo em situações de exceção, recurso valioso (FRENKEL, 2001; BASTOS, 2005).

Certamente a vertente teórica mais afinada com o ambiente de inovação descrito como característico da indústria farmacêutica, em geral, é a de Capacidades Dinâmicas (TEECE; PISANO; SHUEN, 1997; SCHUMPETER, [1934] 1955). Esta escola considera que as fontes de vantagem competitiva são decorrentes de "rendas schumpeterianas", isto é, da competição baseada na inovação, com destruição criativa de competências existentes. As rendas são temporárias e dependem, portanto, de um fluxo incessante de inovações, de produto, processo ou organizacionais. A avaliação das possibilidades a partir desta perspectiva tende a reforçar o paradigma de que o core business das farmacêuticas consiste na pesquisa e desenvolvimento de novas drogas, mas abre ao mesmo tempo espaço para especular sobre novas trajetórias tecnológicas, que poderão resultar em um novo modelo de negócio, ou mesmo em uma nova indústria. A estrutura da indústria farmacêutica, tradicionalmente fundamentada na rota química de desenvolvimento de novas drogas, já está sendo profundamente afetada pela rota da biotecnologia, com novas fronteiras sendo dadas pela genética molecular e combinação de DNA (BASTOS, 2005). Como definir um modelo de negócios para ser bem sucedido no longo prazo? Com as possibilidades de transformação que estão à frente, porque se preocupar com os genéricos? Aos fabricantes de genéricos, fica a pergunta: de onde virão suas rendas no futuro?

\section{As respostas estratégicas: eliminando as fronteiras entre inovação e imitação}

Neste trabalho, propõe-se que as respostas estratégicas das grandes farmacêuticas, e dos grandes competidores de genéricos que surgiram a partir do crescimento expressivo deste 
mercado, tornaram menos claras as separações entre inovação e imitação na indústria. Para desenvolver este argumento, entretanto, será necessário distinguir inovação radical de inovação incremental e esclarecer as definições de paradigmas e trajetórias tecnológicas, bem como situar as grandes farmacêuticas no contexto histórico de inovação em sua indústria.

O conceito proposto por Bastos para inovação radical no contexto da indústria farmacêutica aplica-se, de forma mais ampla e flexível a inovações que: "a) ampliaram o escopo e os mercados de setores existentes por meio da aplicação ou introdução de novos princípios científicos, tecnologias e materiais; b) deslocaram produtos ou processos existentes no mercado e c) serviram como modelos para inovações subseqüentes." (BASTOS, 2005, p.280). Este conceito parece bastante apropriado para a indústria, pois a introdução de inovações radicais não criou ondas de destruição no sentido literal. Basta verificar que as empresas líderes apresentaram grande flexibilidade e adaptabilidade aos diversos choques institucionais e tecnológicos ocorridos; a grande maioria das farmacêuticas de porte está no negócio há mais de um século (ACHILLADELIS; ANTONAKIS, 2001).

Os paradigmas tecnológicos, segundo Dozi (1988), definem contextualmente as necessidades que devem ser atendidas (o problema a solucionar), os princípios científicos (métodos) a serem utilizados para a tarefa e os recursos tecnológicos (artefatos) a serem empregados. As trajetórias tecnológicas estariam, portanto, direcionadas a partir deles, caracterizando-se situações de lock-in e dependência de trajeto (path dependence). A predominância de certos paradigmas na indústria farmacêutica explicaria porque 70\% das inovações mundiais, entre 1800 e 1990, foram introduzidas por apenas 30 empresas, originadas em apenas cinco países (ACHILLADELIS; ANTONAKIS, 2001).

Prosseguindo na linha de raciocínio sugerida por Dozi (1988), podemos constatar que as taxas de inovação expressivas na indústria farmacêutica podem ser explicadas: pelas oportunidades que seus paradigmas propiciam pelo grau de apropriabilidade das inovações (e consequentemente, de seu retorno econômico) e pelos padrões de demanda que enfrenta (no caso da saúde, crescentes e ilimitados). Os paradigmas e trajetórias tecnológicas, contudo, contribuem para um padrão de inovação que, radical ou incremental, permanece sendo gerado (ou apropriado) por um grupo reduzido de grandes corporações.

De fato, a estrutura atual da indústria farmacêutica não difere das características que Pavitt (1984) elencou, em sua taxonomia, como relativas a setores baseados na ciência: as atividades de pesquisa e desenvolvimento são formalizadas e conduzidas em grandes laboratórios; constata-se a existência de um pequeno número de grandes firmas, à exceção de empreendedores "schumpeterianos" ou de firmas altamente especializadas.

Uma outra explicação para esta estrutura encontrada na indústria farmacêutica pode ser dada pelo modelo teórico desenvolvido por Teece (1986): as firmas que já se encontram na indústria podem lucrar com inovações radicais porque detêm ativos complementares importantes, que impedem os inventores de se apropriarem com exclusividade das inovações que desenvolvem; as firmas de biotecnologia, usualmente compradas por ou licenciadoras para grandes farmacêuticas ilustram bem esta situação.

Com o que foi exposto anteriormente, a posição das grandes farmacêuticas orientadas para pesquisa, apesar da ameaça dos genéricos, pode ser considerada ainda como confortável. Isto explicaria porque algumas delas não dedicaram esforços para posicionar-se no mercado de genéricos como Roche, Pfizer, Astra Zeneca, Eli Lilly, Bristol Meyer Squibb.

Percebe-se na indústria farmacêutica, nos últimos anos, uma tendência de crescimento de inovações incrementais. Dentre os novos medicamentos aprovados pelo FDA, entre 1989 e 1994, 46,7\% estavam caracterizados como modificações incrementais, entre 1995 e 2000, esta parcela subiu para 59\% dos registros aprovados (NIHCM, 2002). Este é precisamente o ponto de convergência que se pretende levantar como hipótese neste trabalho. Dois movimentos contrários, por parte de grupos antagônicos, convergindo para um mesmo padrão de inovação: a proliferação de "me too's".

Frenkel (2001) define medicamentos "me too" como aqueles produtos lançados posteriormente ao produto original, com características químicas diferentes, mas atividade terapêutica semelhante. 
Concorrem com o produto inovador, buscando apropriar-se de uma parcela do mercado criado por ele, utilizando-se dos mesmos mecanismos de difusão. Dificilmente entram em concorrência direta de preços com o inovador.

Empresas orientadas para o modelo de inovação podem desenvolver produtos " $m e$ too" como estratégia para conter a investida de genéricos; pequenas modificações em produtos consagrados podem constituir novas patentes ou representar prolongamento da vigência da patente (três anos, no caso do mercado americano). A estratégia se completa com a utilização do marketing farmacêutico para convencer prescritores e compradores quanto aos benefícios do produto modificado, deixando assim o potencial concorrente genérico com um mercado bem menor do que esperava encontrar. Já os grandes fabricantes de genéricos, como a Ranbaxy e a Dr.Reddy's, começaram a investir significativamente em pesquisa e desenvolvimento (PHARMACEUTICAL TECHNOLOGY, 2006), especialmente para desenvolver melhorias incrementais, produtos " $m e$ too", que lhes propiciem patentes próprias e, consequentemente, melhores margens. Naturalmente, para comercializar estes medicamentos de marca, necessitarão de uma estrutura própria ou terceirizada de marketing farmacêutico.

O comportamento dos inovadores e imitadores aqui retratados pode ser caracterizado nos termos do modelo dinâmico de inovação proposto por Tushman-Rosenkopf, segundo o descreve Afuah (1998): descontinuidades tecnológicas provocam uma era de fermentação, finda a qual emerge um design (concepção) dominante em termos de solução tecnológica; o período subseqüente é de mudança incremental, e, no caso de uma indústria com alta complexidade como a farmacêutica, prevaleceria um clima de relativa incerteza, no qual a influência de aspectos que não são de natureza técnica seria elevada; nestas condições, circunstâncias institucionais poderiam vir a influenciar incisivamente a conduta e o padrão das inovações das empresas.

Mas as estratégias empregadas pelas empresas orientadas para inovação não se limitam aos produtos "me too". A criação de uma divisão específica para fabricação de genéricos, como já havia sido teoricamente aventada, mostrou-se a escolha de diversas empresas, notadamente da Novartis, que na busca de liderança neste mercado já empreendeu 8 aquisições de fabricantes de genéricos entre 2000 e 2005. Abbott e Merck também seguiram esta estratégia com aquisições. Como seria de se esperar, em linha com a lógica da busca de liderança em custos, o movimento em termos de fusões e aquisições entre fabricantes de genéricos também foi intenso. Em levantamento da Chemical Market Reporter (2000), 37 operações de consolidação de genéricos foram identificadas no mesmo período mencionado.

Uma outra opção estratégica pode ser observada: as empresas inovadoras, em caso de falta de perspectiva em seus pipelines podem licenciar medicamentos de pequenas empresas, sem estrutura para produzir e comercializar em escala mundial. Os grandes fabricantes de genéricos também podem fazê-lo, buscando ampliar suas linhas de produtos. As motivações podem ser diferentes, mas a conduta estratégica acaba por resultar semelhante.

Finalmente, as empresas inovadoras podem desenvolver estratégias de co-marketing, fazendo o licenciamento de uma molécula patenteada para um segundo produtor. Neste caso, o intuito da empresa inovadora é capturar uma parcela adicional em relação à sua inovação, tendo como exemplo ilustrativo o caso em que se trata de inovação incremental, e a empresa quer conquistar mercado junto a compradores institucionais sensíveis a preço. Os fabricantes de genéricos, paradoxalmente, tornamse opções das farmacêuticas inovadoras na consecução destas estratégias.

As alternativas estratégicas das empresas, orientadas à inovação, que foram aqui apresentadas não são de modo algum originais, mas conhecidas pela indústria (TENDÊNCIAS CONSULTORIA INTEGRADA, 2006a; 2006b). A importância de descrevê-las neste trabalho reside na confirmação de que a separação entre modelos de negócios de inovação e imitação, se em um primeiro momento do mercado de genéricos parecia possível, atualmente está cada vez mais difícil de se estabelecer. Fabricantes de genéricos e empresas orientadas a pesquisa disputam o mesmo mercado. 


\section{CONCLUSÃO}

O presente trabalho debruçou-se sobre o mercado farmacêutico, com intuito de caracterizar a dinâmica de inovação e imitação que pode ser observada a partir da conduta estratégica das empresas envolvidas, tendo como referência inicial a introdução dos medicamentos genéricos. Os genéricos constituem um mecanismo de mercado estimulado no âmbito da legislação nacional por inúmeros países, visando reduzir e/ou conter os gastos com saúde através da redução nos preços dos medicamentos, em função da competição que os genéricos propiciam.

Observou-se que diversas empresas focadas em inovação decidiram competir também com genéricos, criando divisões de negócios cujas fontes de rendas econômicas não mais derivam de inovações. As empresas que se dedicaram desde o início da regulamentação à fabricação de genéricos cresceram extraordinariamente, e voltaram-se também para inovação na busca de fontes de rendas econômicas. Em ambos os casos, as empresas são incentivadas a desenvolver melhorias incrementais que envolvem investimentos e riscos significativamente menores e podem ser utilizados como instrumentos para evitar a competição, em função dos direitos de patentes. Decorridos vários anos da introdução dos medicamentos genéricos nos principais mercados, mundiais tornou-se difícil diferenciar a conduta estratégica das empresas segundo os agrupamentos estabelecidos inicialmente para efeito da análise: inovadores podem ser grandes fabricantes de genéricos e imitadores podem ser reconhecidos como laboratórios inovadores.

Os resultados alcançados neste trabalho sugerem um aprofundamento da análise, em especial no sentido de compreender o impacto e as adaptações que modelos híbridos de inovação e imitação vêm provocando nas empresas farmacêuticas. Tanto a análise, segundo os referenciais de estratégia empresarial, como baseada nos modelos de inovação devem ser refinadas, estabelecendo-se um quadro de referência que possibilite uma compreensão das nuances que, certamente, o alcance deste trabalho não foi capaz de representar.

\section{REFERÊNCIAS}

ACHILLADELIS, B.; ANTONAKIS, N. The dynamics of technological innovation: the case of the pharmaceutical industry. Research Policy, v. 3, n. 30, p. 535-558, Apr. 2001.

AFUAH, A. Innovation management: strategies, implementation and profits. Oxford: Oxford University Press, 1998.

ANVISA. Medicamentos genéricos: informações, notícias, legislação, pesquisas e indicadores. Disponível em: <www.anvisa.gov.br> Acesso em: 14 nov. 2006.

ASSOCIAÇÃO PRÓ-GENÉRICOS. Informações, apresentações, notícias e pesquisas. Disponível em: <www.progenericos.org.br>. Acesso em: 12 nov. 2006.

BARNEY, J. B. Firm resources and sustained competitive advantage. Journal of Management, v. 17, n. 1, p. 99-120, 1991.

BARNEY, J. B. Strategic factor markets. Management Science, v. 32, n. 10, p. 12311241, 1986.

BARTLETT, C. A.; GHOSHAL, S. Going global: lessons from late movers. Harvard Business Review, v. 78, n. 2, p 132-142, Mar./ Apr. 2000.

BASTOS, V. D. Inovação farmacêutica: padrão setorial e perspectivas para o caso brasileiro, BNDES Setorial, n. 19, p. 271-295, set. 2005.

BESANKO, D.; DRANOVE, D.; SHANLEY, M. Economics of strategy. New York: Free Press, 2000.

BRASIL. Lei n. 9.787, de 10 de fevereiro de 1999. Altera a Lei no 6.360, de 23 de setembro de 1976, que dispõe sobre a vigilância sanitária estabelece o medicamento genérico, dispõe sobre a utilização de nomes genéricos em produtos farmacêuticos e dá outras providências. Diário Oficial da União, Brasília, 11 fev. 1999.

BRASIL. Lei 9.279, de 14 de maio de 1996. Regula direitos e obrigações relativos à propriedade industrial. Diário Oficial da União, Brasília, 15 maio 1996. 
BRASIL. Decreto 793, de 05 de abril de 1993. Altera os Decretos n. 74.170 , de 10 de junho de 1974 e 79.094, de 5 de janeiro de 1977, que regulamentam, respectivamente, as Leis n 5.991, de 17 de janeiro de 1973, e 6.360, de 23 de setembro de 1976, e dá outras providências. Diário Oficial da União, Brasília, 6 abr. 1993.

BRASIL. Constituição da República Federativa do Brasil de 1988. Promulgada 05 de outubro de 1988. 1988, publicada no Diário Oficial da União, Brasília, n. 191-A, Brasília, DF, 1988.

CAVES, R. E. Industrial organization, corporate strategy and structure. Journal of Economic Literature, v. 18, n. 1, p. 64-92, Mar. 1980.

CAVES, R. E.; PORTER, M. E. From entry barriers to mobility barriers: conjectural decisions and contrived deterance to new competition. Quarterly Journal of Economics, v. 91, n. 2, p. 242-261, May 1977.

CHEMICAL MARKET REPORTER. Reportagem: Bulking up in generics, v. 15, n. 10 , p. 85-90, 2006. Disponível em: <www.icis.com/Articles/2006/03/11/ $2013007 /$ bulking-up-in-generics.html> Acesso em: 13 Mar. 2006.

CHEMICAL MARKET REPORTER. Reportagem: Waiting for the generics feast, v. 10 , n. 5 , p. $38-43$, 2000. Disponível em: $<$ www.icis.com/Articles/2006/03/11/ $2013007 /$ bulking-up-in-generics.html> . Acesso em: 15 maio 2000.

COSTA, N. R.; SILVA, P. L. B.; RIBEIRO, J. M. Inovações organizacionais e de financiamento: experiências a partir do cenário institucional. In: NEGRI, B.; GIOVANNI, G. (Ed.). Radiografia da saúde no Brasil. Campinas, SP: Unicamp, 2001. p. 291-306.

COMISSÃO PARLAMENTAR DE INQUÉRITO - CPI. Comissão parlamentar de inquérito destinada a investigar os reajustes de preços e a falsificação de medicamentos, materiais hospitalares e insumos de laboratórios. Relatório Final, Brasília, Câmara dos Deputados, Congresso Nacional, Série Ação Parlamentar, 124, p. 279, 2000.
DATAMONITOR. Global pharmaceuticals industry profile, v. 3, p. 112, Aug. 2005a.

DATAMONITOR. Global generics Industry profile, v. 4, p. 122, Aug. 2005b.

DIAS, C. R. C. Medicamentos genéricos no Brasil de 1999 a 2002: análise da legislação, aspectos conjunturais e políticos. 2003. $102 \mathrm{f}$. Dissertação (Mestrado em Saúde Pública) Departamento de Prática de Saúde Pública da Faculdade de Saúde Pública da Universidade de São Paulo, São Paulo, 2003.

DI BATTISTI, P. Gestão estratégica aplicada a dois laboratórios farmacêuticos transnacionais. 234 f. Dissertação (Mestrado em Administração de Empresas) - Escola de Administração de Empresas de São Paulo da Fundação Getúlio Vargas, São Paulo, 2005.

DOZI, G. The nature of the innovative process. In: DOZI, G. et al. (Ed.). Technical change and economic theory. London: Printer Publishers, 1988.

FRANÇA, E. Análise da política nacional de medicamentos no Brasil, 1999 a 2002: o caso dos medicamentos genéricos. 2004. 142 f. Dissertação (Mestrado em Saúde Pública) Departamento de Saúde Coletiva, Centro de Pesquisas Aggeu Magalhães, Fundação Oswaldo Cruz, Recife, 2005.

FRENKEL, J. Análise da cadeia farmacêutica. In: COUTINHO, L. G. et al. Estudo da competitividade de cadeias integradas no Brasil: impacto das zonas de livre comércio. Campinas, SP: Unicamp, 2002.

FRENKEL, J. O Mercado farmacêutico brasileiro: a sua evolução recente, mercados e preços. In: NEGRI, B.; GIOVANNI, G. (Ed.). Radiografia da saúde no Brasil. Campinas, SP: Unicamp, 2001.

IMS HEALTH - GLOBAL PHARMACEUTICAL SALES. 1998 - 2005. Disponível em: <www.imshealth.com>. Acesso em: 10 nov. 2006.

INTERFARMA - ASSOCIAÇÃO DA INDÚSTRIA FARMACÊUTICA DE PESQUISA. INTERFARMA. Para viver mais e melhor. Disponível em: <www.interfarma.com.br>. Acesso em: 12 nov. 2006. 
INTERNATIONAL FEDERATION OF PHARMACEUTICAL MANUFACTURERS ASSOCIATIONS - IFPMA. A plataforma de inovação farmacêutica: sustentar uma saúde melhor para os doentes em todo o mundo. São Paulo: Apifarma, 2004.

MAGALHÃES, L. C. G. et al. Estratégias empresariais de crescimento na indústria farmacêutica brasileira: investimentos, fusões e aquisições, 1988-2002. Brasília: IPEA, 2003.

MARQUES, M. B. Acessibilidade aos medicamentos: o desafio de vincular ciência, tecnologia, inovação e saúde no Brasil. Marília, SP: CGEE; MCT, 2002.

MELLO, M. T. L. Questões de defesa da concorrência no setor farmacêutico. In: NEGRI, B.; GIOVANNI, G. (Ed.). Radiografia da saúde no Brasil. Campinas, SP: Unicamp, 2001.

NEGRI, B.; GIOVANNI, G. Radiografia da saúde no Brasil. Campinas, SP: Unicamp, 2001.

NATIONAL INSTITUTE FOR HEALTH CARE MANAGEMENT RESEARCH AND EDUCATIONAL FOUNDATION - NIHCM. Changing patterns of pharmaceutical innovation. New York: Free Press, 2002.

NISHIJIMA, M. Análise econômica dos medicamentos genéricos no Brasil. 2003. 144 f. Tese (Doutorado em Economia) Faculdade de Economia, Administração e Contabilidade da Universidade de São Paulo, São Paulo, 2003.

ORGANIZAÇÃO MUNDIAL DA SAÚDE OMS. WHO medicines strategy: countries at the core 2004-2007. Washington: Pan American Journal of Public Health, 2004.

PAVITT, K. Patterns of technical change: towards a taxonomy and a theory. Research Policy, v. 13 n. 6, p. 343-374, 1984.

PETERAF, M. A. The cornerstones of competitive advantage: a resource based view. Strategic Management Journal, v. 14 n. 3, p. 179-191, 1993.
PHARMACEUTICAL TECHNOLOGY. Asian producers raise their profiles in the global pharmaceutical value chains. New York: Free Press, 2006.

PHARMACEUTICAL RESEARCH AND MANUFACTURERS OF AMERICA PHRMA. Decade of innovation. New York: Free Press, 2003. Disponível em: <www.phrma.org >. Acesso em: 06 nov. 2006.

PORTER, M. E. Competitive strategy: techniques for analyzing industries and competitors. New York: Free Press, 1980.

Competitive advantage: creating and sustaining superior performance. New York: Free Press, 1985.

QUEIROZ, S.; GONZALES, A. J. V. Mudanças recentes na estrutura produtiva da indústria farmacêutica. In: NEGRI, B.; GIOVANNI, G. (Ed.). Radiografia da saúde no Brasil. Campinas, SP: Unicamp, 2001.

RAMOS-RODRIGUEZ, A. R.; RUÍZNAVARRO, J. Changes in the intellectual structure of strategic management research: a bibliometric study of the Strategic Management Journal, 1980-2000. Strategic Management Journal, v. 25, p. 981-1004, 2004.

REVISTA EXAME. Reportagens sobre o mercado farmacêutico e medicamentos genéricos. Disponível em: <www.portalexame.com.br>. Acesso em: 22 out. 2006.

REVISTA GRUPEMEF. Grupo de Executivos do Mercado Farmacêutico. Reportagens sobre o mercado farmacêutico e de medicamentos genéricos. Disponível em: <www.grupemef.com.br> Acesso em: 25 ago. 2006.

ROMANO, R.; BERNARDO, P. J. B. Padrões de regulação de preços do mercado de medicamentos: experiência brasileira dos anos 90 e novos arranjos institucionais, 1999. In: NEGRI, B.; GIOVANNI, G. (Ed.). Radiografia da saúde no Brasil. Campinas, SP: Unicamp, 2001.

SCHUMPETER, J. A. The theory of economic development: an inquiry into profits, capital, credit, interest and the business cycle. Cambridge: Harvard University, [1934] 1955. 
SILVA, R. A. C. Controle de preços de medicamentos. São Paulo: Estudos FEBRAFARMA, 2004.

TEECE, D. J.; PISANO, G.; SHUEN, A. Dynamic capabilities and stategic management. Strategic Management Journal, v. 18, n. 7, p. 509-533, Aug. 1997.

TEECE, D. J. Profiting from technological innovation: implications for integration, collaboration, licensing and public policy. Research Policy, v. 15, n. 3, p. 285-306, 1986.

TENDÊNCIAS CONSULTORIA INTEGRADA. Tendências setoriais. São Paulo: Banco de Dados Série Históricas, março de 2006a.

TENDÊNCIAS CONSULTORIA INTEGRADA. Tendências setoriais. São Paulo: Banco de Dados Séries Históricas, 2006b.

TRULLEN, J.; STEVENSON, W. B. Strategy and legitimacy. Business \& Society, v. 45 n. 2, p. 178-210, June 2006.

VASCONCELOS, F. C.; CYRINO, A. B. Vantagem competitiva: os modelos teóricos atuais e a convergência entre estratégia e teoria organizacional. Revista de Administração de Empresas, v. 40, n. 4, p. 20-37, out./dez. 2000.

VIEIRA, F. S.; ZUCCHI, P. Diferenças de preços entre medicamentos genéricos e de referência no Brasil. Revista de Saúde Pública, v. 40, n. 3, p. 444-449, 2006.

Recebido: 06/11/2007

Received: 11/06/2007

Aprovado: 02/12/2007

Approved: 23/02/2007 